\title{
A Method to Decrease Exposure to Aerosols for Percutaneous Tracheostomy During the COVID-19 Pandemic
}

\section{Dear Editor,}

The surge of the Coronavirus Disease 2019 (COVID-19) worldwide has resulted in the depletion of intensive care unit (ICU) resources. Up to $17 \%$ of COVID-19 patients require ventilatory support. ${ }^{1,2}$ Early tracheostomy can expedite liberation from and minimise ventilator duration, freeing up resources for the exponential increase in ICU patient numbers.

COVID-19 is spread through droplet transmission. ${ }^{3}$ Barrier methods such as intubating under plastic drapes or in an acrylic box to minimise infection during high risk aerosol generating procedures (AGP) such as intubation and open tracheostomy have been described. ${ }^{4.5}$

We share our experience in performing two bedside percutaneous dilatational tracheostomy (PDT) procedures in the neurosurgical ICU using clear plastic drapes to minimise droplet spread.

\section{Preparation for PDT Procedure}

The indications for the tracheostomies were for airway protection and prolonged ventilation in 2 patients who had depressed consciousness from neurosurgical causes.

For both patients, we confirmed the absence of the severe acute respiratory syndrome coronavirus 2 (SARS-CoV-2) with repeated testing of their endotracheal aspirates before proceeding with PDT.

As we would be inserting the PDT under a plastic drape for the first time, we held a team briefing to ensure that all staff involved understood the processes. Drugs for general anaesthesia, disposable fiberoptic scope and a pre-packed PDT set were brought into the patient's room to minimise staff movement during the tracheostomy. All staff present in the patient's room wore hospital-mandated personal protective equipment (PPE) which consisted of goggles or face shield, N95 masks and full-body, long-sleeved gowns.

A blanket roll was placed under the patient's shoulders to extend his neck which was cleaned and draped. General anaesthesia was induced and atracurium was given for neuromuscular blockade. Suctioning of the trachea was done using the pre-existing in-line suction catheter.
We placed the PDT set on the patient's chest. A large transparent sterile plastic lithotomy sheet, usually used for the spinal or regional procedures in the operating theatre (OT), was placed over the neck and above the PDT set. We used a large clear sterile plastic dressing to occlude the lithotomy hole of the plastic sheet. Withdrawal of the endotracheal tube (ETT) under direct vision, local anaesthetic infiltration, bronchoscopy and serial dilatations, were all performed under the clear plastic sheet. Figure 1 shows how the clear plastic drape acted as an additional barrier to prevent droplet and possibly aerosol spread.

During the first PDT, the plastic drape was not anchored down and kept slipping during the serial dilatations and bronchoscopy. The examination lamp had to be adjusted as light reflection off the plastic drape made visualisation of the surgical field difficult. Technical difficulty during the procedure was increased slightly due to decreased visual acuity through the plastic drape. The proceduralist also had to keep her hands under the sterile drape and prevent unnecessary lifting of the drape.

These issues were addressed during the second PDT a week later on another patient. We anchored the corners of the plastic drape with sterile adhesive tape to prevent movement of the drape. The light source was positioned directly opposite the proceduralist to decrease reflection off the drape.

Although our patients did not have COVID-19 infections, the conditions under which these 2 PDTs were performed simulated the actual conditions which could be adopted for PDT in COVID-19 patients, and helped in refining the technique of operating under a clear drape.

\section{Discussion}

Tracheostomy constituted the majority of procedures in Severe Acute Respiratory Syndrome (SARS) patients during the 2003-2004 pandemic. There is currently no data on the percentage of COVID-19 patients who require tracheostomy, or the best timing for tracheostomy. It is recommended that the timing of 


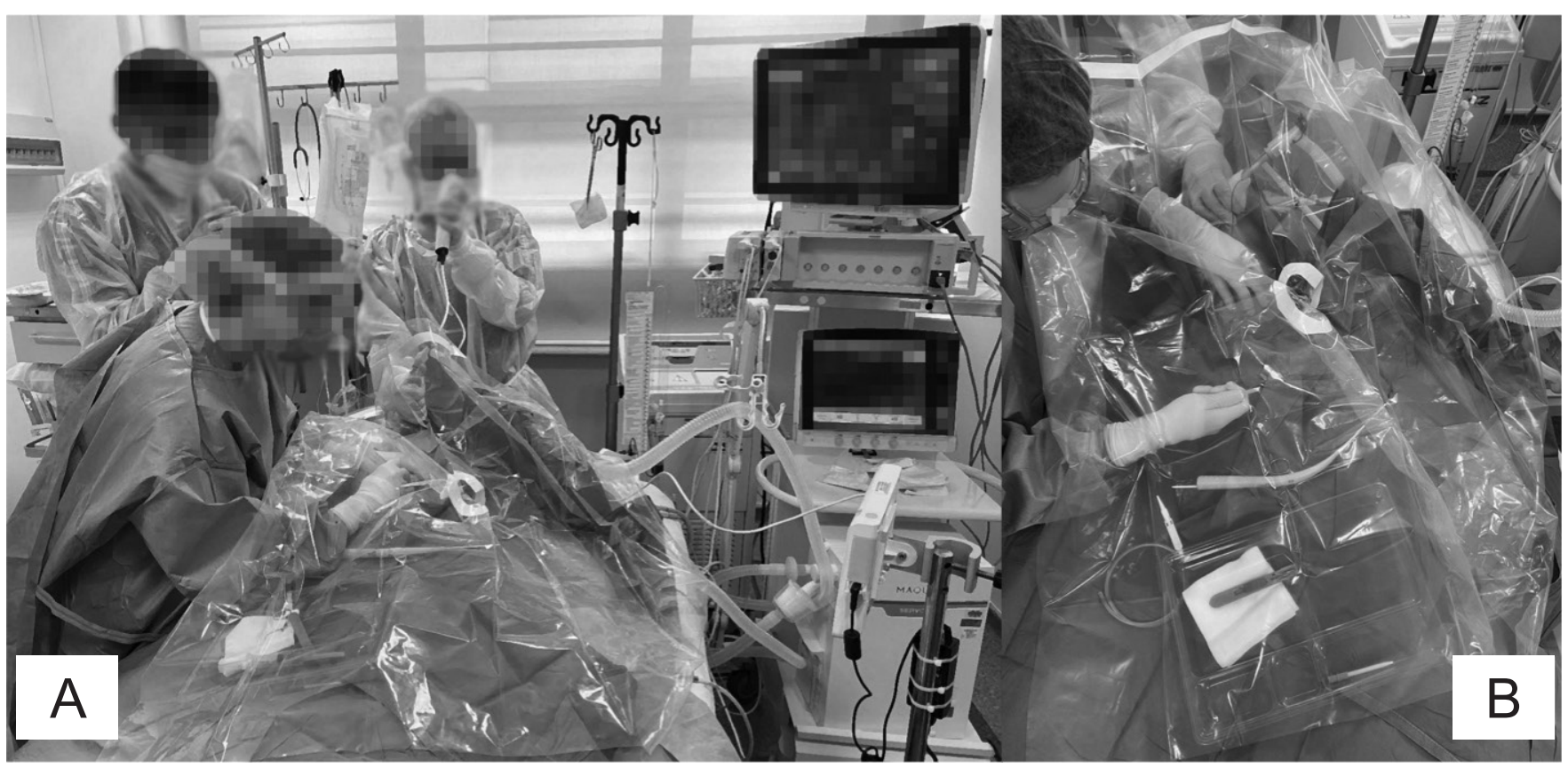

Figure 1. Pictures showing how PDT was performed under a plastic drape. (A) Proceduralist was doing serial dilatation of the trachea while the assistant was performing the bronchoscopy. (B) Closed-up view of the operative fields shows both the hands of the proceduralist and equipment were under the plastic drape.

tracheostomy to be deferred till risk of viral load and shedding is low, after at least 14 days of intubation. ${ }^{6}$

The benefits of PDT over open tracheostomy are reduced stoma site infection and shorter procedure time. ${ }^{7}$ With PDT, there is no need for patient transfer to OT which decreases the morbidity and mortality that may be associated with transfer. ${ }^{8}$

For COVID-19 patients, the greatest benefit of PDT over open tracheostomy is that it can be done as a bedside procedure in a negative-pressure ICU room. This prevents contamination of OT and decreases exposure of healthcare personnel to the infected patient along the transfer route. The same ICU team caring for the patient can perform the PDT without involvement of different staff from the OT team.

A fully disposable tracheostomy set negates the need to wash and sterilise contaminated surgical equipment. Diathermy is not used during the PDT procedure and thus no viral particles-containing vapour plumes are produced.

Overall, this leads to improved resource utilisation time and minimises transmission risk.

There is inconclusive evidence whether PDT or open tracheostomy generates more aerosols. ${ }^{6}$ Bronchoscopy, deflation of ETT cuff and entering the trachea cause unavoidable air leaks in the ventilator circuit. Air leaks can be decreased by using a catheter mount with a bronchoscopy port, and covering this port with a swab can minimise aerosol generation. ${ }^{9}$ An alternative will be using a "closed setup" with a plastic sheath, sealed at both ends, to cover the bronchoscope and the ETT. ${ }^{10}$ Temporary cessation of ventilation during ETT cuff deflation and withdrawal of ETT, hyperinflation of the ETT cuff at least 5-10 $\mathrm{cmH}_{2} \mathrm{O}$ above the baseline cuff pressure to minimise leak, and confirming no or minimal air leak by checking inspiratory and expiratory tidal volumes upon resuming ventilation, help to minimise air leaks. ${ }^{9}$ Ensuring full paralysis with a neuromuscular blocking agent prior to the procedure prevents the patient from coughing.

The alternative of using an acrylic box was considered. One of the main criticisms of using an acrylic box was the impedement to hand movement. Furthermore, for a PDT, a very large and bulky box would be required to allow adequate access.

Stretching a clear plastic drape firmly across an elevated frame above the operative field for better visualisation and stability will help improve the technique. A non-reflective plastic material can be used to minimise light reflection. The tracheostomy set can be placed on a cardiac table under the plastic drape to prevent slippage. Simulation sessions prior to the actual PDT procedure in a COVID-19 positive patient will help to familiarise staff with the new technique. 
The clear plastic drape acts as an additional barrier to minimise droplet spread. Due to limited supply of PPE and supply chain disruptions, staff in some countries have to decontaminate and reuse PPE. ${ }^{11}$ Preventing gross soilage of reusable PPE can reduce viral contamination and prolong the lifespan of PPE.

Due to the overwhelming need for healthcare resources during the current COVID-19 pandemic, minimising infection risk and protecting healthcare workers is a priority. We hope that this simple, cheap barrier method used in addition to standard PPE and powered air-purifying respirators (PAPR) can potentially reduce viral transmission risk during high-risk PDT procedure. ${ }^{11}$

\section{REFERENCES:}

1. Zhou F, Yu T, Du R, Fan G, Liu Y, Liu Z et al. Clinical course and risk factors for mortality of adult inpatients with COVID-19 in Wuhan, China: a retrospective cohort study. Lancet 2020;395:1054-1062.

2. Goh KJ, Choong MC, Cheong EH, Kalimuddin S, Duu Wen S, Phua GC et al. Rapid progression to acute respiratory distress syndrome: review of current understanding of critical illness from COVID-19 infection. Ann Acad Med Singapore 2020;49:108-118.

3. Liu J, Liao X, Qian S, Yuan J, Wang F, Liu Y et al. Community transmission of severe acute respiratory syndrome coronavirus 2, Shenzhen, China, 2020. Emerg. Infect. Dis 2020;26:1320-1323.

4. Matava CT, Yu J, Denning S. Clear plastic drapes may be effective at limiting aerosolization and droplet spray during extubation: implications for COVID-19. Can J Anaesth 2020;67:902-904.

5. Chow VLY, Chan JYW, Ho VWY, Pang SSY, Lee GCC, Wong MMK et al. Tracheostomy during COVID-19 pandemic - novel approach. Head Neck 2020;42:1367-1373.

6. Tracheostomy guidance during the COVID-19 Pandemic. Available athttps://www.entuk.org/tracheostomy-guidance-during-covid19-pandemic. Accessed on 14 April 2020.
7. Klotz R, Probst P, Deininger M, Klaiber U, Grummich K, Diener MK et al. Percutaneous versus surgical strategy for tracheostomy: a systematic review and meta-analysis of perioperative and postoperative complications. Langenbecks Arch Surg 2018;403:137-149.

8. Delaney, A., Bagshaw, S. M., Nalos, M. Percutaneous dilatational tracheostomy versus surgical tracheostomy in critically ill patients: a systematic review and meta-analysis. Crit. Care 2006;10:R55.

9. Takhar A, Walker A, Tricklebank S, Wyncoll D, Hart N, Jacob T et al. Recommendation of a practical guideline for safe tracheostomy during the COVID-19 pandemic. Eur Arch Otorhinolaryngol 2020;277:2173-2184.

10. Wong P, Lim WY, Mok M. Supraglottic airway guided intubation during the COVID-19 pandemic: a closed technique. Anesth Analg. 2020; Online ahead of print.

11. World Health Organization: Rational use of personal protective equipment forcoronavirus disease (COVID-19) and considerations during severe shortages - interim guidance Available from https://apps.who.int/iris/handle/10665/331215. 27 February 2020. Accessed on 17 April 2020.

Michelle Leanne $\underline{\operatorname{Lim}},{ }^{1}{ }_{\text {MBBS, MMed (Anaes), }}$

Irene MJ Wong, ${ }^{1}$ MBBS, MMed (Anaes), EDIC,

Thangavelautham Suhitharan, ${ }^{1}$ MBBS, MMed (Anaes), EDIC,

Andrew S $\underline{\text { Kong, }}{ }^{1}$ MBBS, FRCA, EDIC,

Qing Yuan Goh, ${ }^{1}$ MBBS, MMed (Anaes), EDIC

${ }^{1}$ Department of Surgical Intensive Care, Division of Anaesthesiology and Perioperative Medicine, Singapore General Hospital, Singapore

Address for Correspondence: Dr Qing Yuan Goh, Division of Anesthesiology and Perioperative Sciences, Singapore General Hospital, Outram Road, Singapore 169608

Email: qygoh84@gmail.com 